\author{
A. A. Pypka, N. A. Turbay \\ Oles Honchar Dnipropetrovsk National University
}

\title{
On some generalization of pronormal subgroups in locally finite group
}

We proved that if every subgroup of locally finite group is monopronormal, then this group is a $\bar{T}$-group.

Key words: locally finite group, monopronormal subgroup, contranormal subgroup, Dedekind group, supersoluble group, locally nilpotent residual, $\bar{T}$-group.

Доведено, що локально скінченна група, усі підгрупи якої є монопронормальними підгрупами, буде $\bar{T}$-групою.

Ключові слова: локально скінченна група, монопронормальна підгрупа, контранормальна підгрупа, дедекіндова група, надрозв'язна група, локально нільпотентний резидуал, $\bar{T}$-група.

Доказано, что локально конечная группа, все подгруппы которой являются монопронормальными подгруппами, будет $\bar{T}$-группой.

Ключевые слова: локально конечная группа, монопронормальная подгруппа, контранормальная подгруппа, дедекиндова группа, сверхразрешимая группа, локально нильпотентный резидуал, $\bar{T}$-груnпа. A subgroup $H$ of a group $G$ is said to be pronormal in $G$ if the subgroups $H$ and $H^{g}$ are conjugate in $\left\langle H, H^{g}\right\rangle$ for each element $g \in G$. Well known that normalizer of pronormal subgroup is abnormal. In the same time, every abnormal subgroup is contranormal. Thus, we naturally obtained the following generalization of pronormal subgroups.

Let $G$ be a group and $H$ be a subgroup of $G$. Let $K=\langle H, x\rangle, x \in G$. A subgroup $H$ is called monopronormal in $G$ if for every element $x \in G$ either $H^{x}=H$ or $N_{K}(H)^{K}=$ $K$.

The groups, in which every subgroup is pronormal, have been studied by N.F. Kuzennyj and I.Ya. Subbotin [2]. They proved that if every subgroup of a group $G$ is pronormal, then $G$ is a $\bar{T}$-group. Recall that a group $G$ is said to be a $T$-group, if every subnormal subgroup of $G$ is normal [1]. A group $G$ is said to be a $\bar{T}$-group, if every subgroup of $G$ is a $T$-group [1].

In this connection it is interesting to consider the relationships between monopronormal subgroups and $\bar{T}$-groups. G.

Lemma 1. Let $G$ be a group such that every subgroup of $G$ is monopronormal in

(i) If $H$ is a subgroup of $G$, then every subgroup of $H$ is monopronormal in $H$.

(ii) If $H$ is a normal subgroup of $G$, then every subgroup of factor-group $G / H$ is monopronormal in $G / H$. 


\section{A.A. PYPKA, N.A. TURBAY}

Lemma 2. Let $G$ be a group such that every subgroup of $G$ is monopronormal in $G$. If $H$ is nilpotent subgroup of $G$ then $H$ is a Dedekind group.

Proof. Let $H$ be a nilpotent subgroup of $G$ and $x, y \in H$. Put $K=\langle x, y\rangle$. Since a subgroup $K$ is nilpotent, every subgroup of $K$ is not contranormal, so $N_{K}(H)^{K} \neq K$. Thus $\langle x\rangle^{y}=\langle x\rangle$, which means that any cyclic subgroup of $H$ is normal in $H$. Hence any subgroup of $H$ is normal in $H$. So $H$ is a Dedekind group.

Corollary 1. Let $G$ be a group such that every subgroup of $G$ is monopronormal in $G$. If $H$ is a locally nilpotent subgroup of $G$ then $H$ is Dedekind.

Proof. Let $H$ be a locally nilpotent subgroup of $G$ and $x, y \in H$. Put $K=\langle x, y\rangle$. Since $H$ is a locally nilpotent subgroup, $K$ is nilpotent. By Lemma $2\langle x\rangle^{y}=\langle x\rangle$ for every element $y \in H$. It follows that $H$ is a Dedekind group.

Lemma 3. Let $G$ be a group such that every subgroup of $G$ is monopronormal in $G$. Let $H$ be a subgroup of $G$ and $A$ be a normal abelian subgroup of $H$. Then every subgroup of $A$ is $G$-invariant.

Proof. Let $H$ be a subgroup of $G$ and $A$ be a normal abelian subgroup of $H$. Let $a \in A$. If $A=\langle a\rangle$ then the proof is obvious. Let $\langle a\rangle$ be a proper subgroup of $A$ (i.e. $\langle a\rangle \neq A)$. Put $K=\langle A, y\rangle$, where $y \in H \backslash A$. Obviously in this case $A \leq N_{K}(\langle a\rangle)$ and factor-group $K / A$ is abelian. It follows that $K \unrhd N_{K}(\langle a\rangle)$. Thus $N_{K}(\langle a\rangle)=K$. So every proper cyclic subgroup $\langle a\rangle$ is $H$-invariant and then $G$-invariant, since $H$ is arbitrary subgroup of $G$. Hence every subgroup of $A$ is $G$-invariant.

Corollary 2. Let $G$ be a group such that every subgroup of $G$ is monopronormal in $G$. Let $H$ be a nilpotent subgroup of $G$ and $A$ be a minimal abelian $H$-invariant subgroup of $H$. Then $A$ is a cyclic subgroup of prime order.

Lemma 4. Let $G$ be a finite group such that every subgroup of $G$ is monopronormal in $G$. Then $G$ is supersoluble.

Proof. Let $S_{p}$ be a Sylow $p$-subgroup of $G$, where $p$ is a least prime number dividing the order of group $G$. Put $L=N_{G}\left(S_{p}\right)$. By Corollary $2 S_{p}$ has a chief series, whose factors are cyclic of prime order. Denote the chief factor of subgroup $S_{p}$ as $A / B$. The order of factor-group $L / C_{L}(A / B)$ divides $p-1$. But $p$ is least and we have $C_{L}(A / B)=$ $N_{G}\left(S_{p}\right)=L$. So chief factor $A / B$ is central. Hence $N_{G}\left(S_{p}\right)=S_{p} \times S_{p^{\prime}}$. If $p>2$ then $S_{p}$ is abelian and $S_{p} \leq \zeta\left(N_{G}\left(S_{p}\right)\right)$. Then by Burnside's Theorem $S_{p}$ has a normal complement in $G$. If $p=2$ then by Lemma $2 S_{2}$ is Dedekind and by Theorem 1 from [3] $S_{2}$ also has a normal complement in $G$. So in both cases there is a normal subgroup $H$ such that $G=H \lambda S_{p}$. Further, using similar arguments we choose Sylow $q$-subgroup $S_{q}$ in $H$, where $q$ is a least prime number dividing the order of $H$. So $S_{q}$ has a normal complement $K$ in $H$, i.e. $H=K \lambda S_{q}$. Continuing this process we obtain Sylow series such that all its factors are cyclic of prime order. It follows that group $G$ is supersoluble. 
Corollary 3. Let $G$ be a locally finite group such that every subgroup of $G$ is monopronormal in $G$. Then $G$ is a locally supersoluble.

Proof. Let $H$ be a finitely generated subgroup of $G$. Since $G$ is a locally finite, $H$ is finite. Note that every subgroup of $H$ is monopronormal in $H$. By Lemma 4 a subgroup $H$ is supersoluble. Thus every finitely generated subgroup of $G$ is supersoluble. Hence $G$ is locally supersoluble.

Theorem 1. [4] Let $G$ be a finite group and $A$ be an abelian normal subgroup of $G$. If the factor-group $G / C_{G}(A)$ is nilpotent then $A=Z \times E$, where all chief factors of $Z$ are central and all chief factors of $E$ are eccentric.

In this case we say that a subgroup $A$ has a $Z$-decomposition in $G$.

Lemma 5. Let $G$ be a locally finite group such that every subgroup of $G$ is monopronormal in $G$. Then all $G$-chief factors of locally nilpotent residual $L$ is eccentric.

Proof. Suppose that locally nilpotent residual $L$ has a central factors. Let $A$ be an abelian normal subgroup of locally nilpotent residual $L$. Denote by $\mathfrak{S}$ the set of all finite subgroups of $G$. Let $H \in \mathfrak{S}$ and suppose that $\mathfrak{H}$ is a set of all finite $H$-invariant subgroups of $A$. Let $B \in \mathfrak{H}$. By Theorem 1 a subgroup $B$ has a $Z$-decomposition: $B=Z_{B} \times E_{B}$, where all chief factors of $Z_{B}$ are central and all chief factors of $E_{B}$ are eccentric. Note that if $B \leq C$, where $C \in \mathfrak{S}$, then $Z_{B} \geq Z_{C}, E_{B} \leq E_{C}$. Put $E=\bigcup_{H \in \mathfrak{S}} E_{H}$. So $E$ is a normal subgroup of $G$. All chief factors of $E$ are eccentric and all chief factors of the factor-group $A / E$ are central. Thus $A / E \leq \zeta_{\infty}(G / E)$. The factor-group $G / A$ is locally nilpotent, then $G / E$ is also locally nilpotent. A subgroup $E$ is a proper subgroup of $A$, i.e. $A \neq E$. On the other hand $G / E$ is nilpotent, hence $A \leq E$. So we obtain a contradiction. Thus $L$ doesn't has central factors.

Theorem 2. Let $G$ be a locally finite group such that every subgroup of $G$ is monopronormal in $G$. Then $G$ is a $\bar{T}$-group.

Proof. Since $G$ is locally finite group such that every subgroup of $G$ is monopronormal in $G$, by Corollary $3 G$ is locally supersoluble. This means that $G$ has a Sylow descending series (no necessarily finite)

$$
G=G_{0} \geq G_{1} \geq G_{2} \geq \ldots,
$$

where $G / G_{1}$ is a Sylow $p_{1}$-subgroup, $G_{1} / G_{2}$ is a Sylow $p_{2}$-subgroup etc., in addition $p_{1}<p_{2}<\ldots$ In particular, the factor-group $G / G_{1}$ can be a 2-group. Let $L$ be a locally nilpotent residual of group $G$. Since $G / G_{1}$ is a locally nilpotent 2-group then $G_{1} \geq L$, and then $2 \notin \Pi(L)$. Thus Sylow $p$-subgroups of $L$ are abelian.

Since $G$ is supersoluble, the derived subgroup $[G, G]$ is locally nilpotent. So $L$ is also locally nilpotent because $[G, G]$ contains $L$. Since $2 \notin \Pi(L), L$ is abelian.

The factor-group $G / L$ is locally nilpotent, then by Corollary 1 it is Dedekind. A subgroup $L$ is abelian and normal in $G$, then by Lemma 3 every subgroup of $L$ is $G$-invariant. 
By [1] it remains to prove that $\Pi(L) \cap \Pi(G / L)=\emptyset$. Assume that it is not true. Let there is a prime number $p$, such that $p \in \Pi(L) \cap \Pi(G / L)$. Note that $p \in \Pi(L)$ and $2 \notin \Pi(L)$, so $p \neq 2$. Since $L$ is abelian, $L=S_{p} \times S_{p^{\prime}}$. Put $H=G / S_{p^{\prime}}$ and $K=L / H$. Then factor-group $L / H$ is a $p$-subgroup and $H / K$ is nilpotent. Let $P$ be a Sylow $p$ subgroup of $H / K$ (i.e. $P \in S y l_{p}(H / K)$ ). Note that $P$ contains $K$ and $\zeta(P / K) \neq\langle 1\rangle$ because $H / K$ is nilpotent. By Lemma 5 all $G$-chief factors of nilpotent residual $L$ are eccentric. Put $Z=\langle z K\rangle \leq \zeta(P / K)$. Since factor-group $P / K$ is central, $[H, Z] \leq K$. By Theorem 1 and last inclusion $Z=C \times K$, where $C$ is a some subgroup. On the other hand, by Lemma 3 every subgroup of $Z$ is $G$-invariant. Pick the element $x \in \Omega_{1}(K)$, where $\Omega_{1}(K)$ is the lower layer of $K$, i.e. $\Omega_{1}(K)=\{x \in K|| x \mid=p\}$. So there is an integer $k$ such that $k$ is relatively prime to $p$ and $x^{g}=x^{k}, g \in G$. Pick an element $c$ of $C$. Then $c^{g}=c$. So $(x c)^{g}=x^{g} c^{g}=x^{k} c \neq(x c)^{s}$ for some $s$. So we obtain a contradiction. It shows that $Z$ contains an element which is not $G$-invariant. Hence $G$ is a $\bar{T}$-group.

Note that Theorem 2 can't be generalized to the case of arbitrary periodic groups. The following example illustrates this.

Example 1. Let $G$ be a Tarski monster group, i.e. $G$ is infinite group which proper non-identity subgroups are finite cyclic of prime order $p$. Note that A. Yu. Ol'shanskii proved the existence of such groups for any prime $p>10^{75}[5, \S 28]$. In $G$ every element generates cyclic subgroup of prime order $p$ and every two non-commuting elements generate the whole group $G$. If $y \in\langle x\rangle$, then $\langle x\rangle^{y}=\langle x\rangle$. If $y \notin\langle x\rangle$, then $\langle x, y\rangle=G$ and $\langle x\rangle=N_{G}(\langle x\rangle)$. Thus $N_{G}(\langle x\rangle)^{G}=G$. So $G$ is the periodic group such that every subgroup of $G$ is monopronormal in $G$, but $G$ isn't a $\bar{T}$-group.

\section{References}

1. Robinson D.J.S. Groups in which normality is a transitive relation/ D.J.S. Robinson // Proc. Cambridge Philos. Soc. 60 (1964).-- P.21-38.

2. Subbotin I.Ya. Locally soluble groups in which all infinite subgroups are pronormal/ I.Ya. Subbotin, N.F. Kuzenny // Izvestiya VUZ, Math. 32 (1988).- P.126-131.

3. Ballester-Bolinches A. Sylow permutable subnormal subgroups of finite groups/ A. Ballester-Bolinches, R. Esteban-Romero// Journal of Algebra 251 (2002).- P.727738.

4. Baer R. Durch Formationen bestimmte Zerlegungen von Normalteilern endlicher Gruppen/ R. Baer // Journal of Algebra 20 (1972).- P.38-56.

5. Ol'shanskii A. Yu. Geometry of defining relations in groups / A. Yu. Ol'shanskii // Mathematics and its Applications, 1991.- 505 p. 